\title{
Teaching physics and understanding infrared thermal imaging
}

\section{Michael Vollmer, Klaus-Peter Möllmann}

Michael Vollmer, Klaus-Peter Möllmann, "Teaching physics and understanding infrared thermal imaging ," Proc. SPIE 10452, 14th Conference on Education and Training in Optics and Photonics: ETOP 2017, 104522C (16 August 2017); doi: 10.1117/12.2266142

SDIE Event: 14th Conference on Education and Training in Optics and Photonics, ETOP 2017, 2017, Hangzhou, China 


\title{
Teaching physics and understanding infrared thermal imaging
}

\author{
Michael Vollmer, Klaus-Peter Möllmann, \\ University of Applied Sciences Brandenburg, 14770 Brandenburg/Germany
}

\begin{abstract}
Infrared thermal imaging is a very rapidly evolving field. The latest trends are small smartphone IR camera accessories, making infrared imaging a widespread and well-known consumer product. Applications range from medical diagnosis methods via building inspections and industrial predictive maintenance etc. also to visualization in the natural sciences. Infrared cameras do allow qualitative imaging and visualization but also quantitative measurements of the surface temperatures of objects. On the one hand, they are a particularly suitable tool to teach optics and radiation physics and many selected topics in different fields of physics, on the other hand there is an increasing need of engineers and physicists who understand these complex state of the art photonics systems. Therefore students must also learn and understand the physics underlying these systems.
\end{abstract}

Keywords: optics education, infrared imaging, thermography, visualization, IR technologies

\section{1) INTRODUCTION}

The story of infrared imaging started in 1800, when Herschel discovered infrared radiation experimentally at long wavelengths just outside the visible spectrum of sun light. The quantitative explanation of incandescent infrared radiation in 1900 by Max Planck started a development, which today has resulted in modern infrared technologies with infrared camera systems These are also the result of scientific developments in semiconductor physics and micro-system technologies. Nowadays, infrared imaging has numerous applications, such as failure analysis, leak detection or in general predictive maintenance in various industries. Topics range from gas detection in the petrochemical industry to building envelope inspections, study of electrical appliances, solar panels and many more. Besides, IR imaging provides an excellent instrument for teaching physics by qualitative visualization and/or quantitative analysis of a great number of physical phenomena. These involving e.g. detection of minute energy transfer, e.g., processes due to friction, heat transfer, electrical power dissipation or optics and radiation laws. Here, special emphasis is on the use of IR imaging at university level education in lectures, student projects and laboratory courses.

\section{2) BASICS OF NIR IMAGING}

Every object at a temperature $\mathrm{T}>0 \mathrm{~K}$ emits radiation. Similar to visible light, this thermal radiation is electromagnetic radiation, however, at longer wavelengths. The spectra of thermal radiation can be calculated from Planck's law for incandescent radiation, i.e. black bodies (e.g. [1-3]). Typical temperatures range from $0{ }^{\circ} \mathrm{C}$ to $1000{ }^{\circ} \mathrm{C}$; consequently, thermal radiation - if plotted versus wavelength — is located in the wavelength region from 1 to $20 \mu \mathrm{m}$ (see Fig. 1). Real objects such as walls, skin, glass, paints etc., emit less radiation than black bodies. The ratio of the actual emission with respect to the black body emission is called emissivity $\varepsilon$.

For most practical applications, $\varepsilon$ which may depend on temperature, material and surface properties of the objects as well as on wavelength is a constant. In this case, one speaks of a grey body (for more information on selective emitters for which $\varepsilon$ is wavelength dependent, see [1]). Figure 2 depicts schematically the different emissivities and respective emitted thermal radiation from black bodies, grey bodies and selective emitters. For opaque objects, emissivity $\varepsilon$ is simply related to reflectivity $\mathrm{R}$ via $\varepsilon=1-\mathrm{R}$.

14th Conference on Education and Training in Optics and Photonics: ETOP 2017, edited by Xu Liu, Xi-Cheng Zhang, Proc. of SPIE Vol. 10452, 104522C · (c) 2017 ICO, IEEE, OSA, SPIE CCC code: $0277-786 X / 17 / \$ 18 \cdot$ doi: $10.1117 / 12.2266142$ 


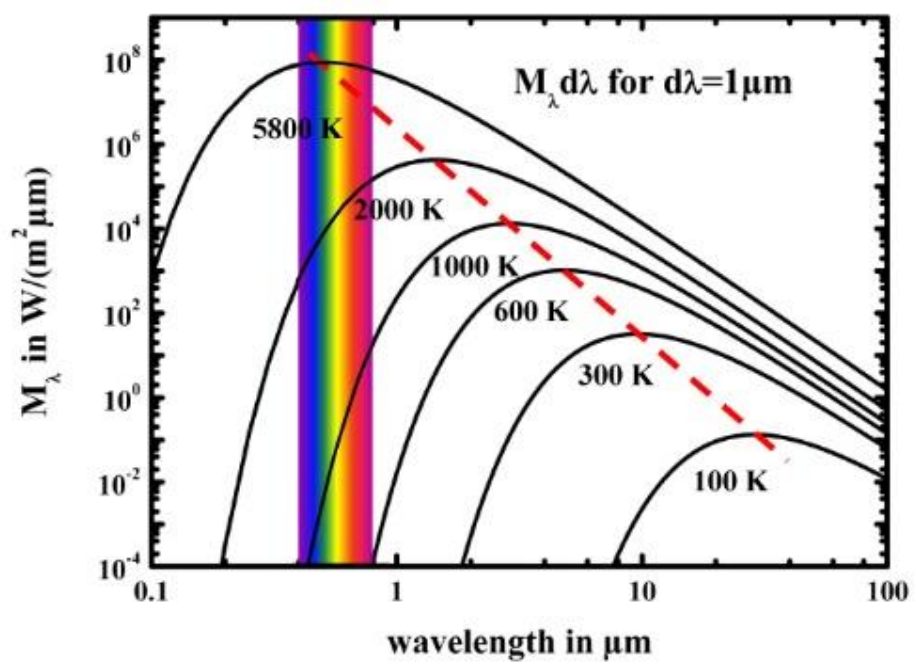

Fig. 1: Excitance spectra of black body radiation for different temperatures. For objects with typical temperatures below $2000 \mathrm{~K}$, the emission is predominantly in the thermal IR range between $1 \mu \mathrm{m}$ and around $20 \mu \mathrm{m}$ wavelength. The dotted line connects all maxima and visualizes Wien's displacement law.
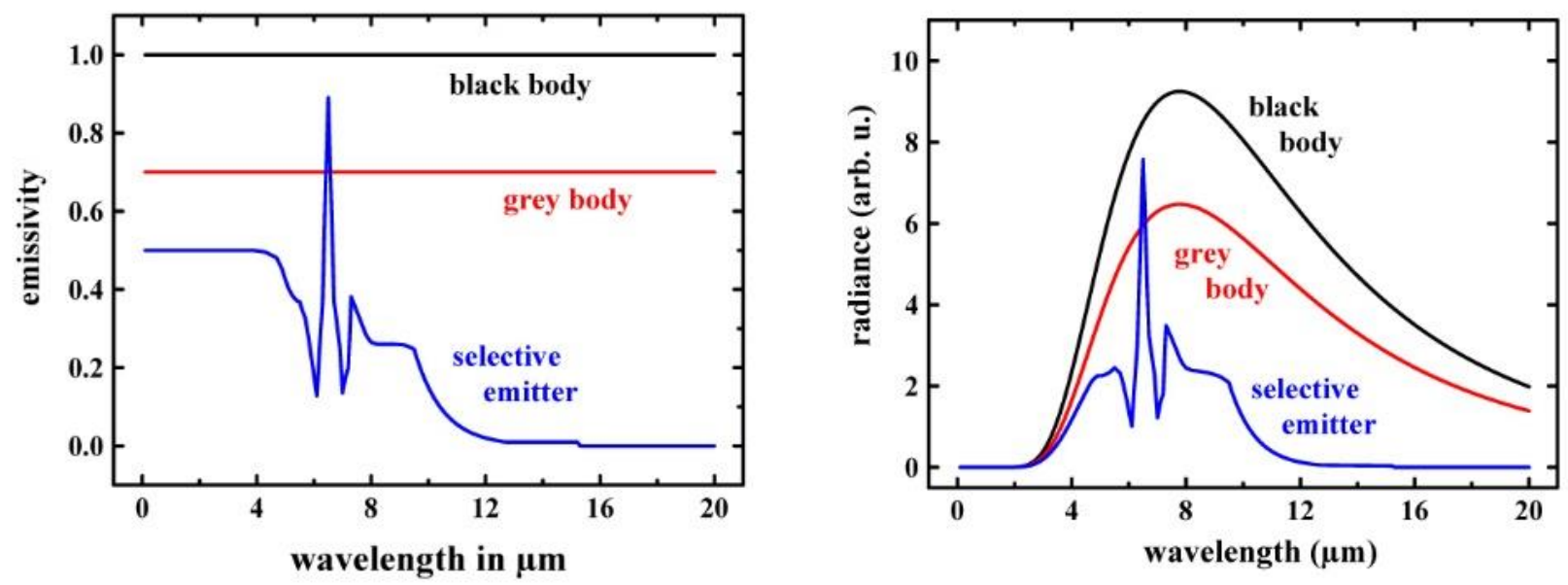

Fig. 2: Emissivities (left) and resulting radiance spectra (right) of thermal radiation for objects of $\mathrm{T}=373 \mathrm{~K}\left(100{ }^{\circ} \mathrm{C}\right)$. The emissivity may be unity (black body), constant (grey body) or vary as a function of wavelength (selective emitter).

Infrared imaging uses only a small portion of the spectrum between $\lambda=1 \mu \mathrm{m}$ and $20 \mu \mathrm{m}$. The restriction is due to several factors, the most important being the transmission spectrum of the atmosphere. Most objects are observed through the atmosphere and some of its constituents, in particular the greenhouse gases $\mathrm{H}_{2} \mathrm{O}$ and $\mathrm{CO}_{2}$ can absorb part of the thermal infrared radiation due to ro-vibrational excitations. Figure 3 depicts the transmission spectrum of a $10 \mathrm{mpath}$ of atmospheric air with $50 \%$ relative humidity at $300 \mathrm{~K}$ and a $\mathrm{CO}_{2}$ concentration of $380 \mathrm{ppm}$ (nowadays, the $\mathrm{CO}_{2}$ concentration has already passed the $400 \mathrm{ppm}$ value, however, the spectrum of $400 \mathrm{ppm}$ is mostly identical with very small changes at $\lambda \approx 4.2 \mu \mathrm{m}$ and $\lambda>15 \mu \mathrm{m}$. 


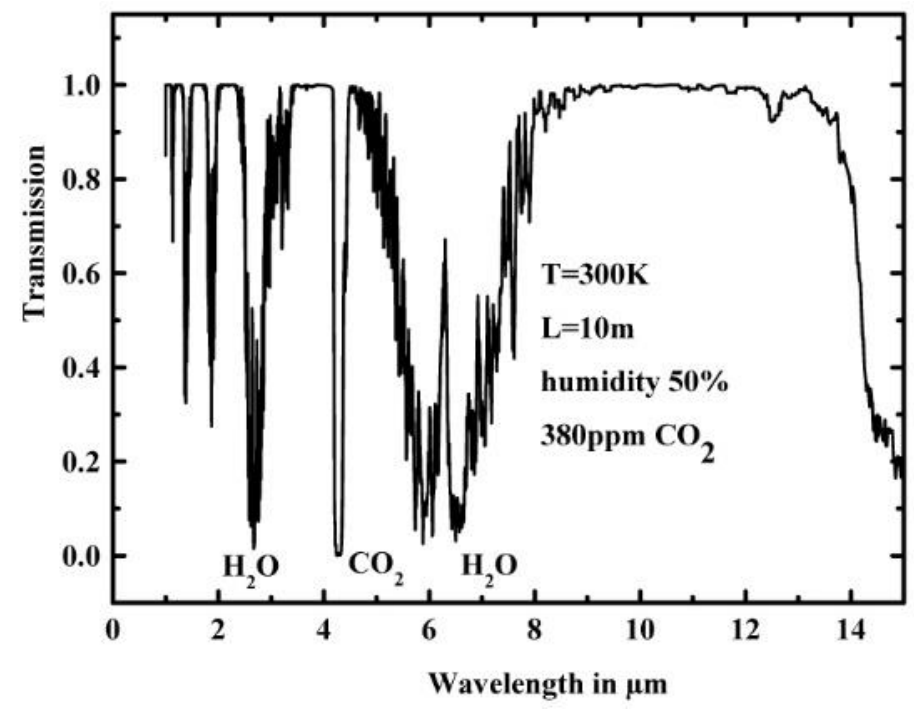

Figure 3. Atmospheric transmission (low resolution) for a standard atmosphere at $\mathrm{T}=300 \mathrm{~K}$, a path length of $10 \mathrm{~m}, \mathrm{CO}_{2}$ concentration of $380 \mathrm{ppm}$ and $50 \%$ relative humidity.

Obviously, there are regions with strong absorption. They separate the so-called atmospheric windows which are nowadays used for infrared thermal imaging. Today commercial cameras are available mostly for the three wavelength regions called longwave (LW) infrared from $8 \mu \mathrm{m}$ to $14 \mu \mathrm{m}$, mid-wave (MW) infrared from around $3 \mu \mathrm{m}$ to $5 \mu \mathrm{m}$, and short-wave (SW) infrared from around $0.9 \mu \mathrm{m}$ to $1.7 \mu \mathrm{m}$. The $\mathrm{MW}$ and SW bands include some narrow spectral absorption features, however, one may correct for these for any quantitative measurement.

Infrared thermal imaging quantitatively measures thermal radiation with infrared sensitive detectors to generate images. Modern detectors consist of focal plane arrays quite similar to CCD or CMOS detector arrays of regular digital cameras. The main difference to VIS light detectors is that quantitative measurements are performed, leading to more expensive systems. Various types of thermal and photoelectric detectors exist. The current standard quality commercial IR cameras have sensor arrays with usually 640 x 512 pixels, i.e. much less than any regular VIS light camera.

Recently, more expensive advanced megapixel detector arrays have become available. On the other hand, less expensive systems with, e.g., $120 \times 160$ pixels or even less for the smartphone IR camera accessories for a few hundred dollars are available.

\section{3) SELECTED EXAMPLES}

Besides applications in industry, infrared cameras are a particularly suitable tool to teach optics and radiation physics and many selected topics in different fields of physics (see [1,4-6] and refs. therein). In addition, there is an increasing need of engineers and physicists who need to understand these complex state of the art photonics systems for their professional careers. Therefore, we developed a course on infrared technologies within an advanced Bachelor program as well as a Master program at our university. Topics within the course include basics such as radiation laws, detector technologies and characterization, optics as well as applications in various fields. Besides infrared imaging, the course also focuses on infrared spectroscopy. In addition, we use infrared imaging also as visualization tool within other lectures to demonstrate physics principles and phenomena. Here we present a few selected examples mostly in a qualitative descriptive manner in order to illustrate the large variety of potential topics. Much more detailed information on each topic can be found in a standard textbook [1]. 
IR images are almost exclusively displayed as false color scale images. In the following examples we want to give an overview of what may be done with IR imaging. Therefore we often suppress the quantit ative temperature scale. More information on quantitative imaging see [1].

\section{1) Friction}

Mechanical phenomena in physics often involve frictional forces. As a consequence part of kinetic energy within a system is transferred into heat. Fig. 4 depicts an example of a motorcycle which is breaking with blocked tires. The tire slides on the ground surface and the frictional forces lead to a transient heating up of both contact areas, i.e. the tire as well as the $g$ round. The snapshot was recorded shortly after stopping the bicycle and after the rear wheel was turned by another $90^{\circ}$ or so in order to also show the hot spot on the former ground contact area on the tire.

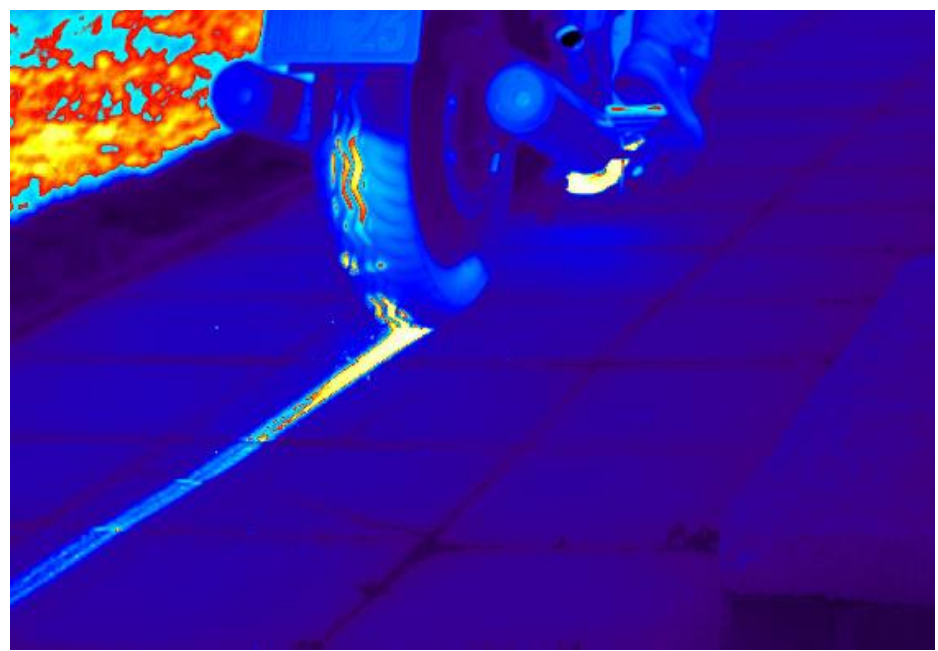

Fig. 4: Friction results in heat transfer from the tire of a motorcycle to the ground upon breaking with blocked tire.

\section{2) Thermal phenomena: convection and heat transfer}

Infrared thermal imaging measures mostly surface temperatures of opaque objects. Therefore the method is obviously very suitable when illustrating thermal physics phenomena. We present two examples, here. First, Fig. 5 illustrates Newton's law of cooling for objects of different size [7]. Four painted metal cubes of sizes between $20 \mathrm{~mm}$ and $60 \mathrm{~mm}$ were heated to the same initial starting temperature within an oven. The cooling was then recorded with IR imaging. As expected from simple heat transfer equations, smaller objects cool down much more rapidly than larger objects.
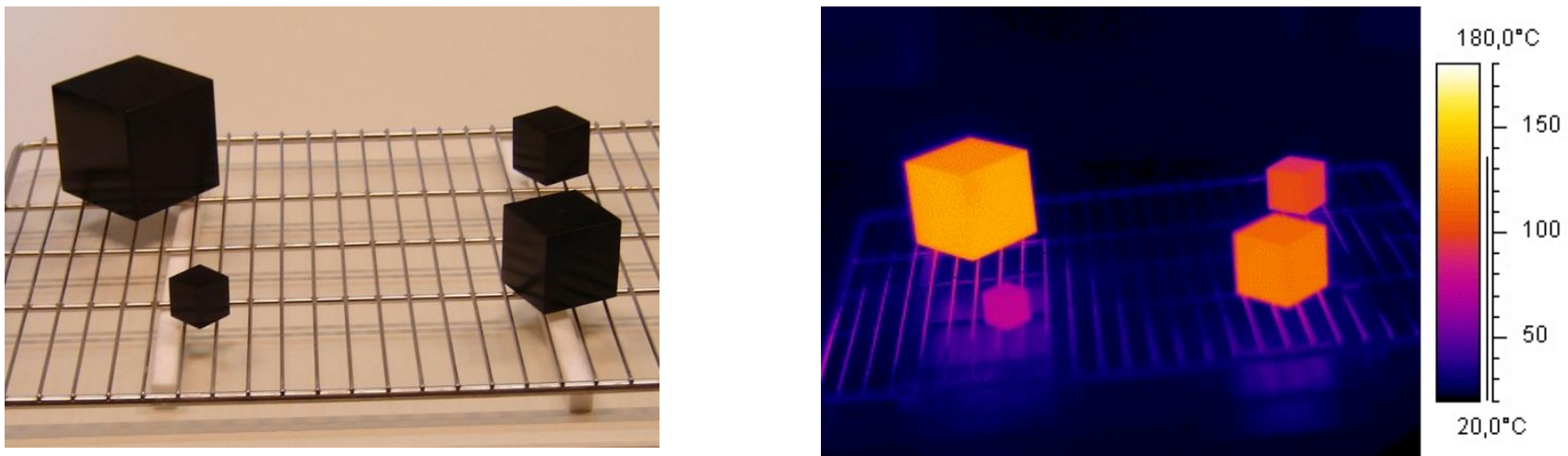

Fig. 5: Cooling of cubes of various sizes which were homogeneously heated to the same starting temperature occurs via heat transfer. The resulting law of cooling leads to faster cooling of small objects. 
Second, Fig. 6 shows the thermal convection patterns within a glass beaker filled with water and a small ice cube floating on top and slowly moving around.

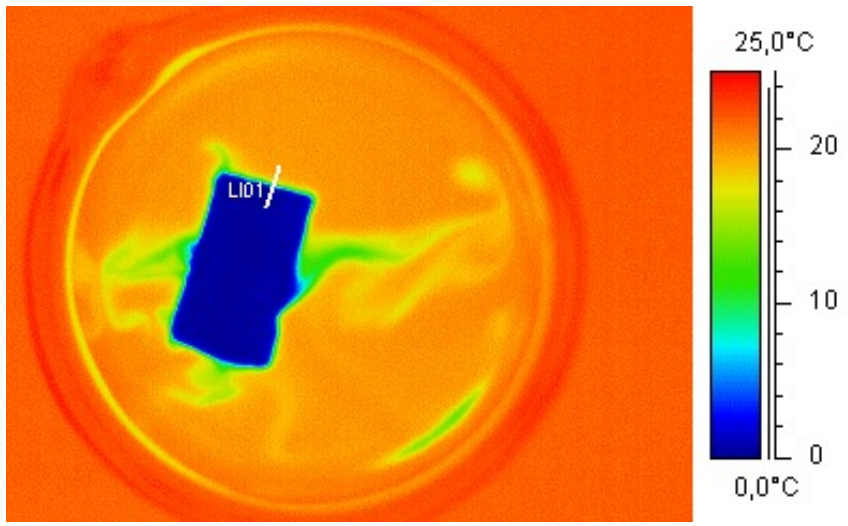

Fig. 6: An ice cube moving in room temperature water observed from above does nicely visualize convective heat transfer

\section{3) Optics: material properties}

Many materials exhibit pronounced differences when observed in the thermal infrared region of the spectrum compared to their appearance in the visible spectral range. For example, un-doped silicon wafers are opaque for VIS radiation but transparent for wavelengths above $1.1 \mu \mathrm{m}$, i.e. IR cameras can just look through wafers. Similarly there are materials which are transparent in the VIS range and opaque in the IR or they are transparent in both ranges. Fig. 7 depicts a respective example of a person wearing special glasses. The right hand side is made of regular glass which transmits visible radiation but absorbs thermal IR wavelengths above around $3 \mu \mathrm{m}$ wavelength and therefore appears opaque. The left hand side is made of $\mathrm{NaCl}$ which is transparent in both spectral ranges.
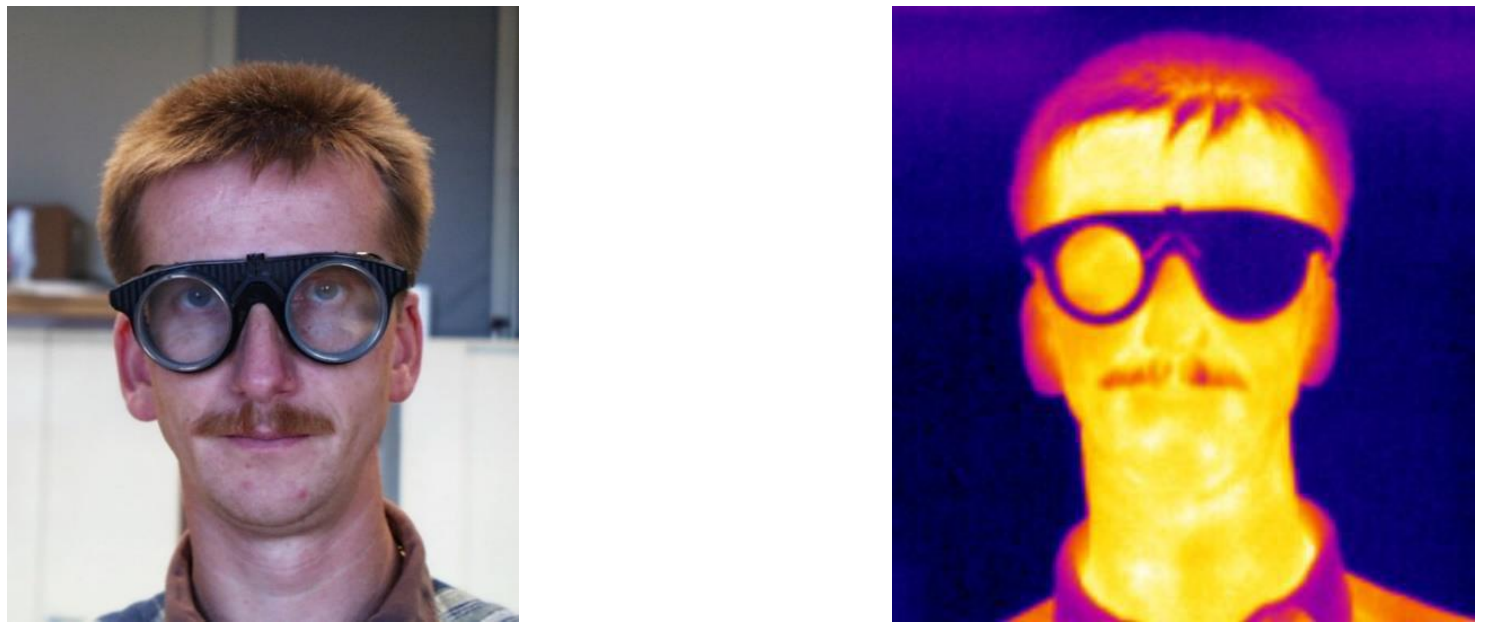

Fig. 7: Special glasses made from glass and $\mathrm{NaCl}$ visualize the different optical material properties in the VIS and IR.

Wafers, glass or $\mathrm{NaCl}$ are materials which are either transparent or opaque. In terms of emissivity they reflect grey objects, i.e. their reflectivity and therefore also their emissivity do not vary within a well-defined spectral region. Most plastics, however, are made of hydrocarbon compounds. Their transmission spectra within the thermal infrared spectral region are characterized by many potential vibrational excitations, i.e. complex line spectra with many absorption features, but there are also spectral regions with high transmission. 
As a consequence, it is usually quite easy to look through plastics using IR cameras. Fig. 8 shows an example for selective emitters. For demonstration purposes, a person has some grey color plastic bag above his head. Visually it is not possible to detect the face, in the thermal infrared, however, one may easily see the face contours below the bag. It is even possible to see that the person is wearing regular glasses.
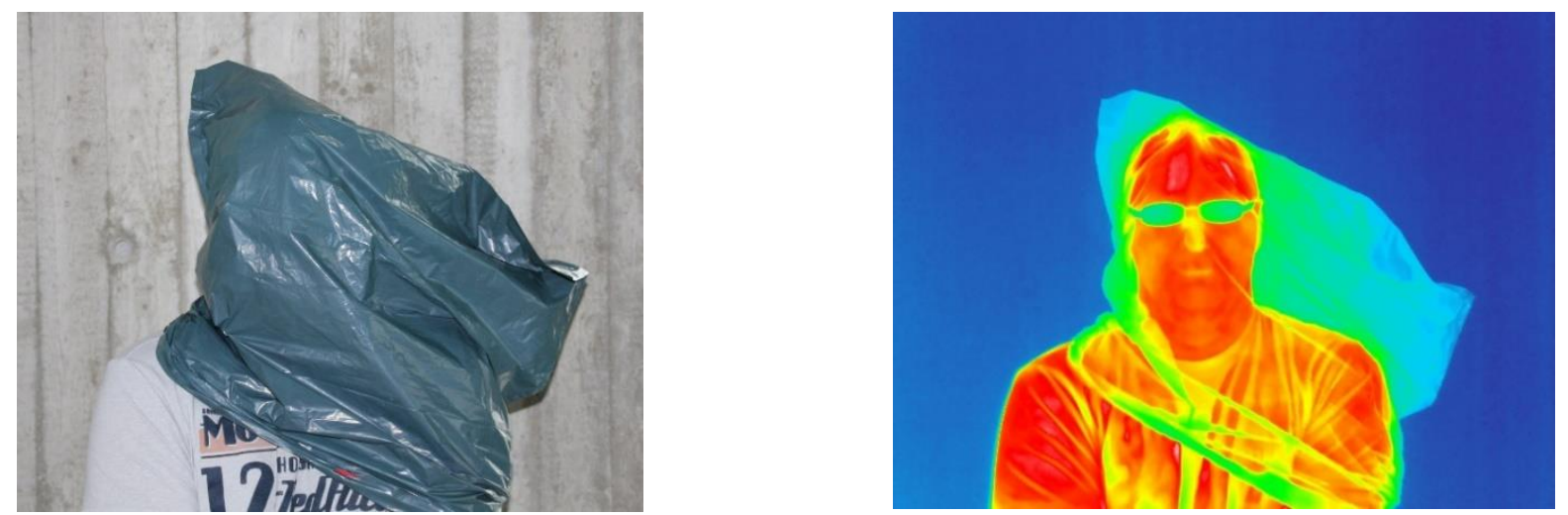

Figure 8: Plastic bags are partially transparent to IR radiation (which may also be quantified using IR spectroscopy)

\section{4) Radiation laws}

In most experimental conditions in thermal physics, one has to deal with nonequilibrium conditions. An instructive experiment uses a small cavity, which resembles some kind of blackbody radiator. Here we use small graphite cylinders with an additional hole in the center of a side wall. Such graphite tubes are standard sample holders in atomic absorption spectroscopy. It is easily possible to heat the cavity by holding the end between fingers $\left(T>30^{\circ} \mathrm{C}\right)$. After a dynamic thermal equilibrium between fingers and cavity is established, it is warmer than the surrounding, that is, the cavity is now not in thermal equilibrium with the colder surroundings. According to the laws of radiation, the temperature difference between cavity and surroundings will lead to a net emission of thermal radiation. Since the cavity has a higher value of emissivity than the side wall, it emits more radiation as clearly shown in Figure 9a.

The situation may also be reversed by cooling the cavity. This was done by placing it between two ice cubes. After a (dynamic) thermal equilibrium between ice cube and tube is established, the tube and its cavity are now much colder than the surrounding at room temperature. The cavity itself has high emissivity, that is, also high absorptivity and it will therefore absorb more radiation from the surrounding than the surface of the graphite tube (more details, see [1]).
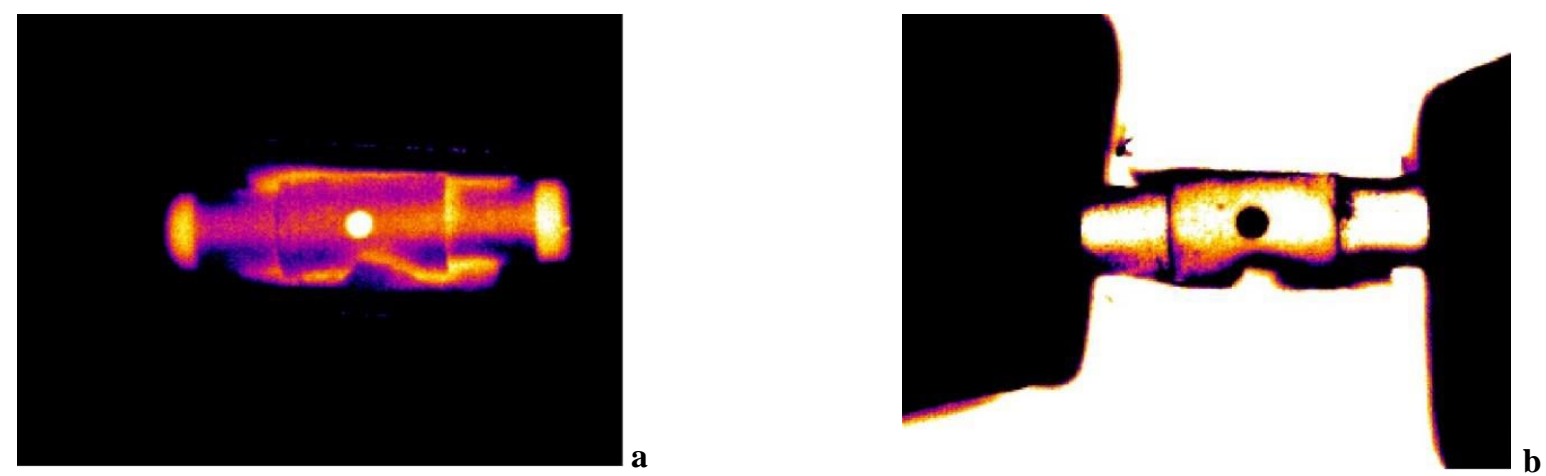

Fig. 9 A graphite tube (length $=3 \mathrm{~cm}$ ) with a small hole (diameter $=2 \mathrm{~mm}$ ) serves as cavity, used to record IR images while studying the transition from emission to absorption. The tube can be heated by holding its ends between fingers (a) or cooled (b) by attaching the ends to ice cubes. 


\section{5) Gas detection}

For many years, the importance of gases in IR imaging was restricted to being the medium through which the IR radiation of objects was transmitted before reaching the detector of the camera. Therefore gases should neither absorb nor emit any IR radiation in order not to change the camera signals. These restrictions for the gas encountered in most applications, that is , atmospheric air, defined the typical spectral ranges of IR.

In contrast, the qualitative detection of gases themselves by commercial thermal imaging is a rather new application. The respective IR cameras utilize absorption or emission of IR radiation in ro-vibrational bands of molecules contributing in the thermal IR region between 1 and $15 \mu \mathrm{m}$. Although strong absorption/emission features allow detection of gases with broadband IR cameras operating in the MW and LW range, more sensitive commercial cameras use narrowband cold filters in front of the detector. At present, commercial gas detecting cameras are available for the detection of many volatile organic compounds (VOCs) including refrigerants, $\mathrm{SF}_{6}, \mathrm{CO}$ and most recently $\mathrm{CO}_{2}$. Fig. 10 depicts two examples which illus trate gas detection for well defined $\mathrm{CO}_{2}$ flows in front of a warm object. In addition to just visualizing gases, one may use the technique to demonstrate different gas flow regimes, here the transition from laminar into turbulent flow.
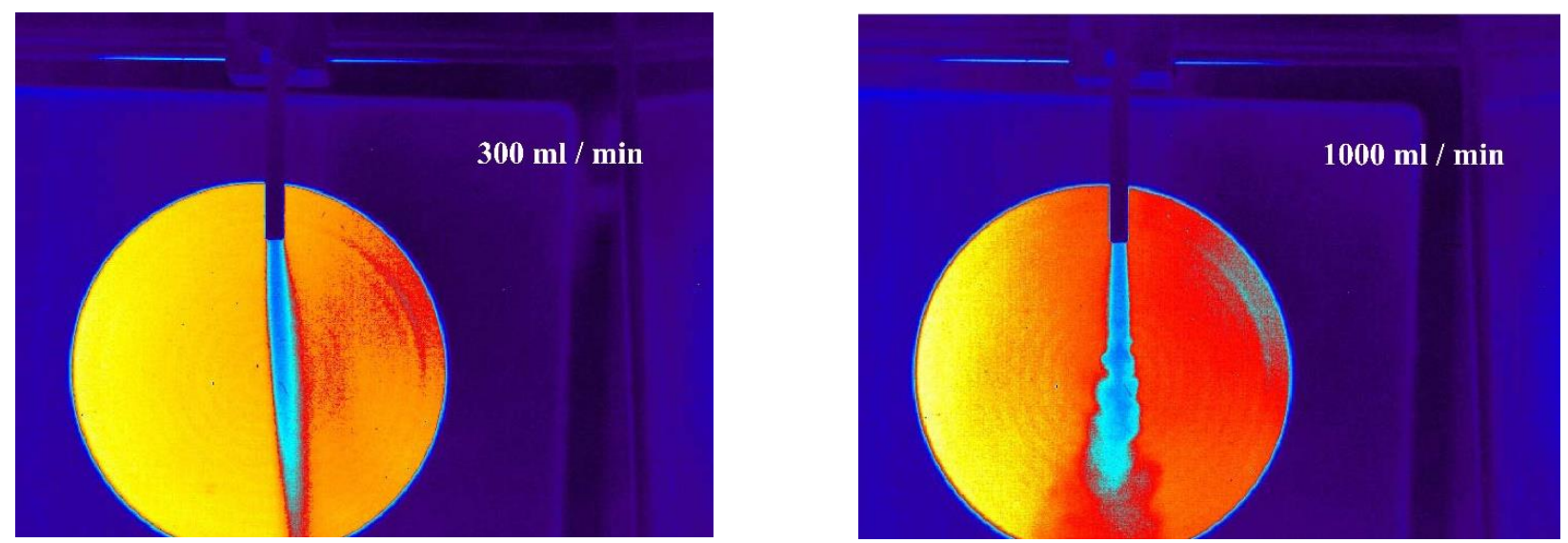

Fig. 10 Raw data of detected $\mathrm{CO}_{2}$ flows of $300 \mathrm{ml} / \mathrm{min}$ and $1000 \mathrm{ml} / \mathrm{min}$. The MW IR camera was operated with an uncooled narrow band filter in front of a black body emitter operated at $T=50^{\circ} \mathrm{C}$.

\section{6) Hidden structures: cars and building inspections}

Quite often, one has to deal with the problem of hidden structures below homogenously appearing surfaces. Various techniques are available to non-destructively test such structures.
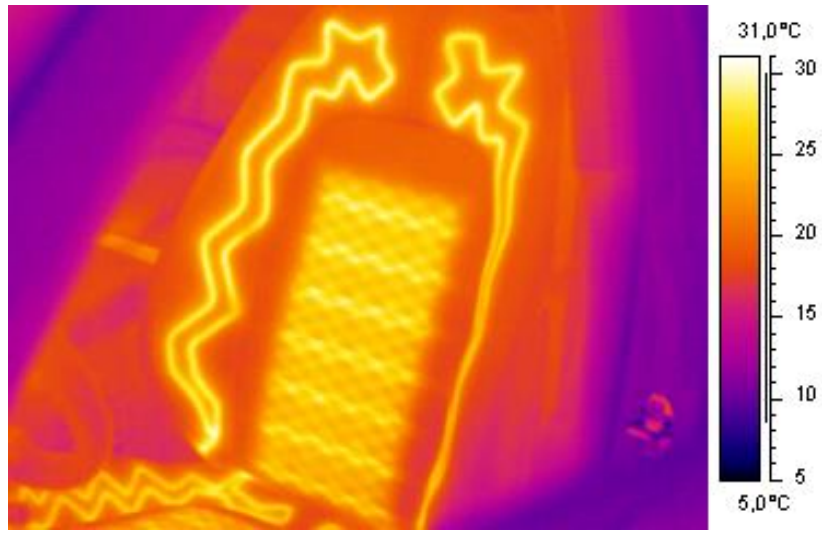

Fig. 11: Visualization of proper operation of a front seat heating system in a car. 
Fig. 11 depicts an example of a front seaterheater in a car. IR imaging can nicely visualize the hidden heater and may therefore e.g. be used as quality control technique for heaters of seats or wind screen s etc. in the car industry.
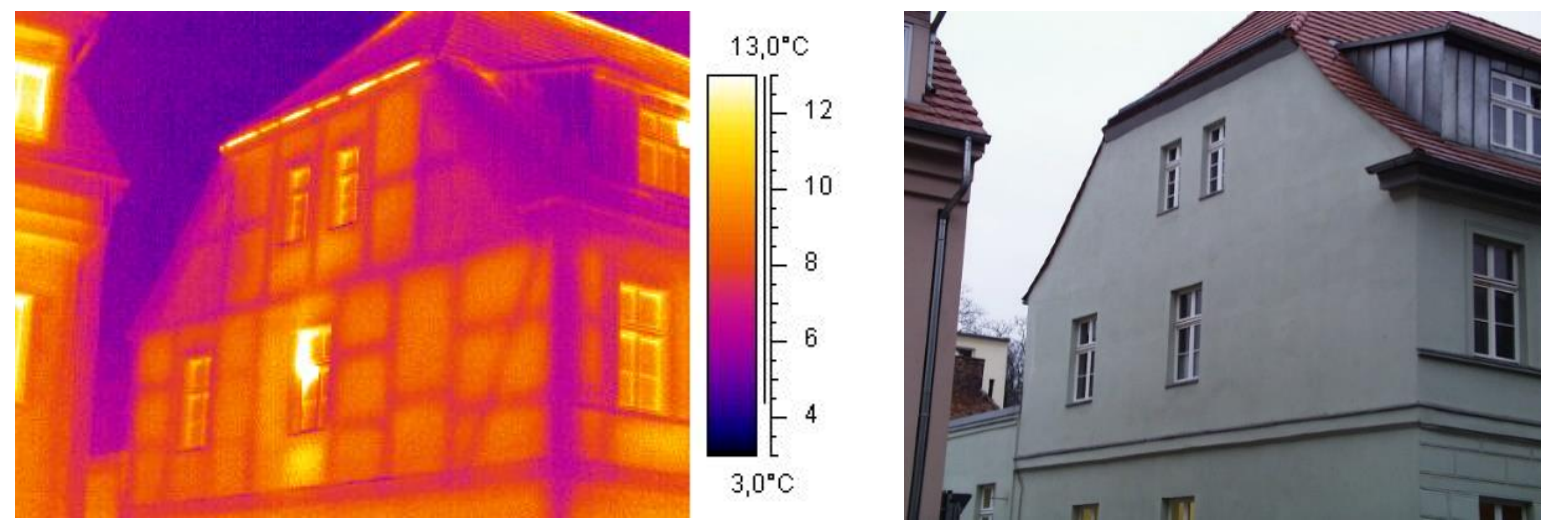

Fig. 12: Half-timbered structures of a house which are hidden behind plaster.

Another example of hidden structures concerns the probably most well-known application of thermal imaging which is building thermography. It is based on differences in thermal properties of different materials if large temperature gradients between inside and outside exist. Whenever the thermal insulation of a building is poor, there will be an energy flow from the warm inside of a house to the cold outside and any structural defects or missing insulation is easily detected. Depending on the thermal conductivities and heat capacities of the wall materials, certain parts will show variations of the surface temperature distribution. These differences are most pronounced for half timbered structures which have per se regular construction patterns. Fig. 12 shows a respective example.

\section{7) Security}

Not surprisingly, IR thermal imaging has a lot of applications in the field of security and surveillance. IR imaging is used extensively for the protection of critical infrastructure, that is, industrial as sets such as airports, power plants, petroch emical installations, etc. In particular, it is used for perimeter monitoring. Figure 13 depicts a typical example while monitoring a dark parking lot.

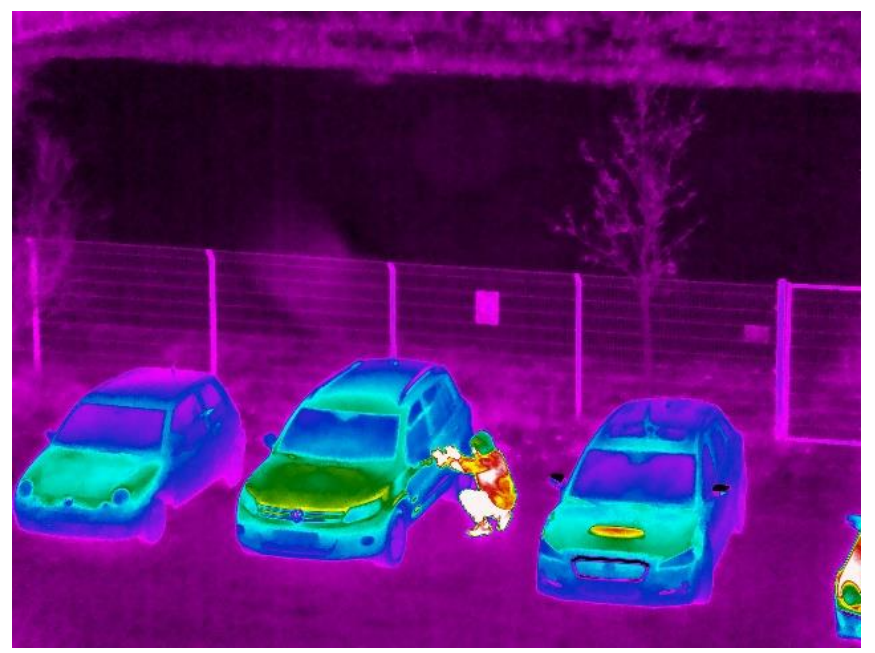

Fig. 13 Monitoring a secured parking lot during the night with a surveillance camera. Attempts of car theft can be detected. 


\section{8) Sports}

Ball games in sports often involve collisions with energy transfer from kinetic energy into heat. An example is tennis. Here IR imaging may even be used to determine whether a service was in or out. Figure 14 depicts two snapshots of a high speed IR imaging sequence of a tennis ball hitting the floor and shortly afterwards while leaving it again. On both images, the contact areas of the racket with the ball can be seen as a grid like structure. In addition, the floor has heated up after the collision. The bottom figure displays measured temperature increases of the floor contact spot as a function of time. For services with about $200 \mathrm{~km} / \mathrm{h}$, temperature increases of more than $10 \mathrm{~K}$ occur, decaying over a period of more than $10 \mathrm{~s}$.
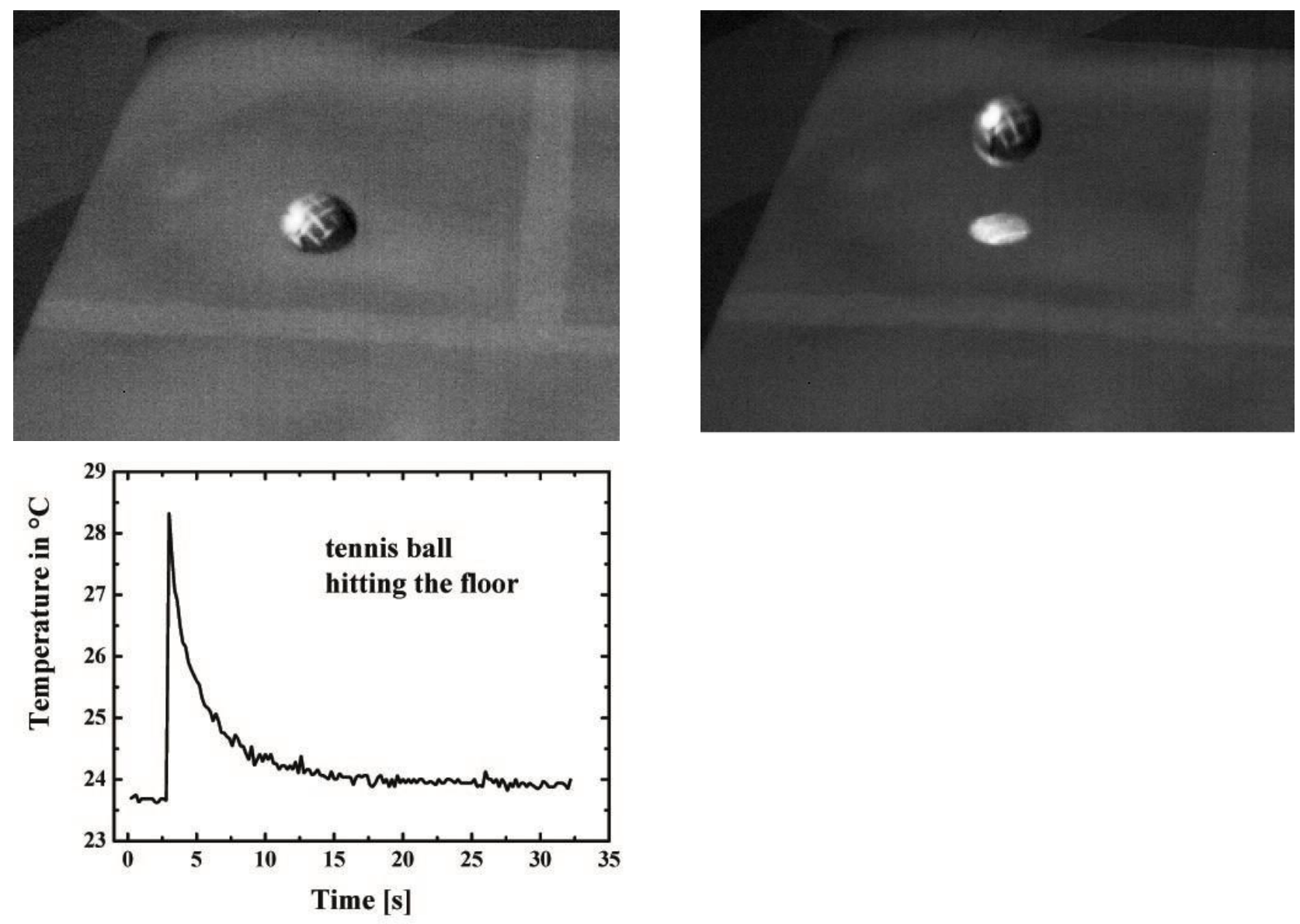

Fig. 14: Two snapshots of a high speed IR imaging sequence of a tennis ball hitting the floor and shortly afterwards while leaving it again as well as a plot of maximu m temperature of the contact spot on the floor versus time.

\section{9) Phenomena in nature}

Having access to an IR camera as a physicist usually means that it is directed towards anything interesting. Outdoors, this may, e.g., be sun or moon. A potential problem, encountered for IR imaging studies of Sun or Moon is the spatial resolution. Older cameras with $320 \times 240$ pixels - even if equipped with $10^{\circ}$ FOV lenses - would only give around 15 pixels across the $0.5^{\circ}$ diameter of Sun or Moon. This may just be sufficient to get a measure from the central pixel, but does not resemble a nice image. The following IR image of the moon (Fig. 15a) was recorded using a radiometrically calibrated Photon $640 \mathrm{LW}$ camera (640x 512m pixels) combined with a $200 \mathrm{~mm}$ telescope optics. This resulted in about 100 pixels across the disk of the moon. 
The average surface temperature of the moon is due to the thermal equilibrium between the solar irradiance and the emitted thermal radiation. As a result, the expected maximu m lunar temperatures range around $394 \mathrm{~K}\left(121^{\circ} \mathrm{C}\right)$ in the center which is easily accessible to any commercial camera. Due to the reduced irradiance towards the edge, the temperature decreases steeply. At full moon, LW IR images show a very symmetrical radiance, i.e. also a symmetric temperature distribution across its disk which is nearly in accordance with the theoretically expected one (Fig. 15c) after taking into account potential atmospheric attenuation due to aerosol layers [8].
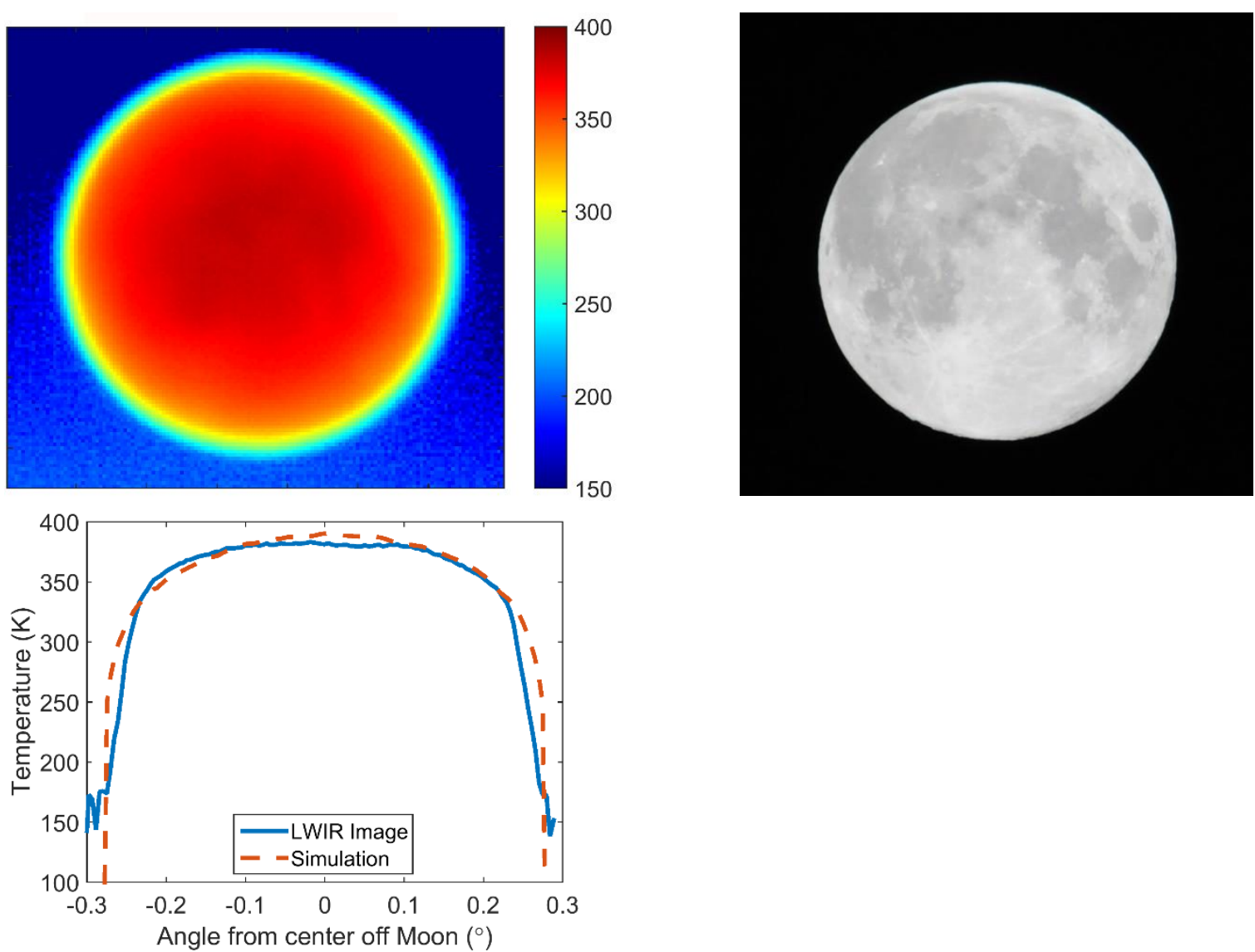

Fig. 15 LW Infrared (a) and VIS (b) images of moon from August 2 ${ }^{\text {nd }}, 2012,1.7 \mathrm{~h}$ after full moon, lunar elevation $30^{\circ}$. Temperatures are in Kelvin. (c) horizontal line profile of measurement (blue line) and simulation (red dotted line) (Images: courtesy Joseph A. Shaw, Paul W. Nugent, MSU Bozeman).

Geothermal phenomena belong not only to the most spectacularbut also to the most dangerous phenomena in nature. A safe observation of geothermal physics is possible in National Parks such as Yellowstone in the USA or in Iceland, where spectaculargeysereruptions taking place at well-defined time intervals. Besides Geysers, hot springs and pools belong to the most fascinating natural thermal phenomena [9]. Being connected to underground thermal reservoirs, they keep very high temperatures all year long. They are usually very colorful since thermopiles may grow on the hot pool walls, depending on temperature. Combined with residual scattering within the extremely clear water, colors spanning the complete visible spectral range result [10]. Fig. 16 depicts a panorama view of the world's third largest hot pool, Grand Prismatic Spring in Yellowstone National Park. Its extensions are about $75 \mathrm{mx} 115 \mathrm{~m}$ with a maximu m depth of around 50m. The average surface temperatures vary across the seasons typically between $63^{\circ} \mathrm{C}$ and $74^{\circ} \mathrm{C}$. One can readily see the hot outflows as well 
as thermal reflections of the boardwalk with tourists. The hot steam feature in the background is due to the much smaller Excelsior Geyser. For more examples of infrared imaging within Yellowstone National Park, see [9].

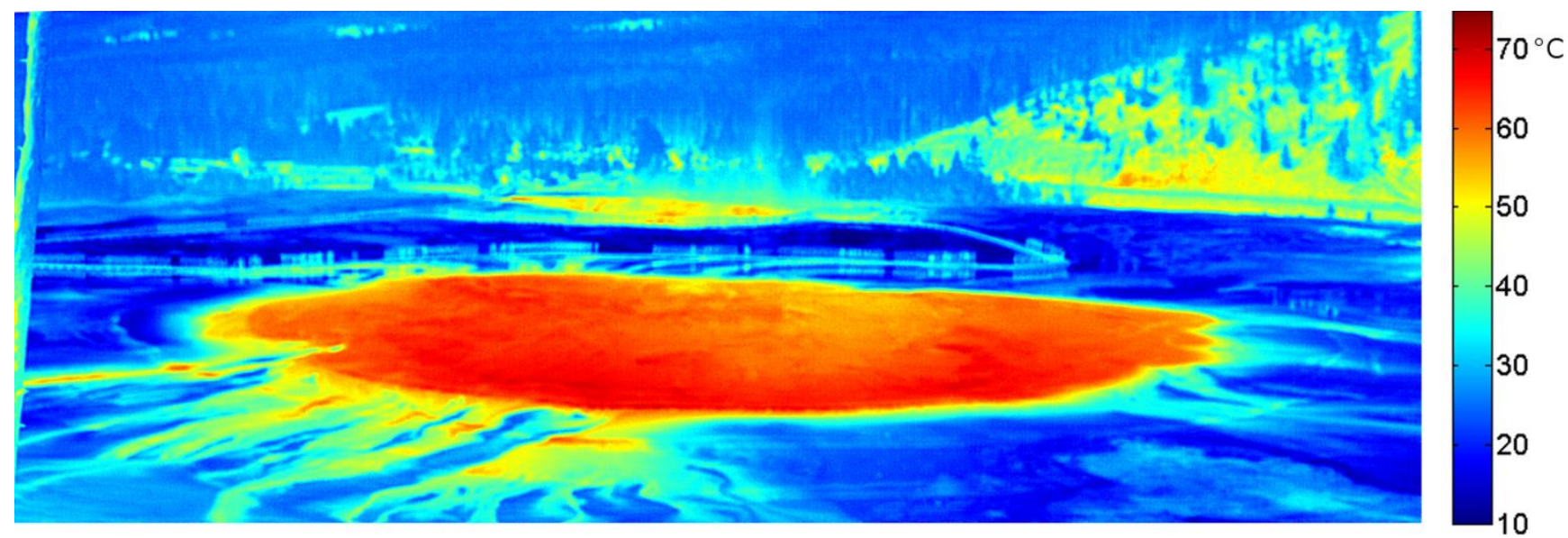

Fig. 16: Image composite of Grand Prismatic Spring in YNP (Image courtesy: Joseph A. Shaw, Paul W. Nugent, MSU Bozeman)

\section{Acknowledgements:}

Projects related to Figs. 15 and 16 were realized during sabbatical stays at MSU Bozeman/Mt in 2012 and 2016 . One of us (MV) gratefully wants to thank his host Prof. Joseph A. Shaw from MSU for his support and Dr. Paul W. Nugent (MSU) for participating in respective experiments and data analysis.

\section{5) REFERENCES}

[1] Möllmann, K.-P. and Vollmer, M., "Infrared Thermal Imaging: Fundamentals, Research and Applications ," Wiley (2010), also available in Spanish and Korean, completely revised and extended $2^{\text {nd }}$ ed. in print

[2] Wolfe, W.L. and Zis sis, G.J., "The Infrared Handbook" 4th printing, revised edition. The Infrared Information Analysis Center, Environmental Research Institute of Michigan / USA (1993)

[3] DeWitt D.P. and Nutter G.D., "Theory and Practice of Radiation Thermometry" , Wiley, New York (1989)

[4] Karstädt, D., Möllmann, K.-P., F. Pinno, F. and Vollmer, M., There is more to see than eyes can detect: visualization of energy transfer processes and the laws of radiation for physics education, The Physics Teacher 39, 371-376 (September 2001)

[5] Möllmann, K.-P. and Vollmer, M., "Infrared thermal imaging as a tool in university physics education," Eur. J. Phys. 28, S37-S50 (2007).

[6] Möllmann, K.-P. and Vollmer, M., “Characterization ofIR cameras in student labs”, Eur. J. Phys. 34/6, S73-90 (2013)

[7] Vollmer, M., "Newton's law of cooling revisited", Eur. J. Phys. 30, 1063-1084 (2009)

[8] Shaw, J.A., Nugent, P.W., Vollmer, M., "Infrared moon imaging for remote sensing of atmospheric smoke layers", Applied Optics 54/4, B64-B75 (2015)

[9] Shaw, J.A., Nugent, P.W., Harris, W., and Vollmer, M., Infrared Yellowstone, Optics and Photonics News (OPN) June 2017, p. 36-43

[10] Shaw, J.A., Nugent, P.W., Vollmer, M., “Colors of thermal pools at Yellowstone National Park", Applied Optics 54/4, B128-B139 (2015) 